\title{
Manifestaciones bucales en pacientes pediátricos con asma. Estudio de Casos Controles
}

$\begin{array}{r}\text { Brizuely Abigail Domenzain Sánchez, } \\ \text { Martha Gabriela Chuc Gamboa, } \\ \text { Fernando Javier Aguilar Pérez, } \\ \text { Alicia Leonor Pinzón Te, } \\ \text { Marina Eduviges Rejón Peraza, } \\ \text { Vicente Esparza Villalpando, } \\ \text { (D) } \\ \hline\end{array}$

\section{Resumen}

El asma afecta en mayoría a la población infantil de 3 a 14 años. Los infantes que padecen enfermedades respiratorias tienden a sufrir alteraciones en su salud bucal. Objetivo: Establecer las manifestaciones clínicas bucales de pacientes pediátricos con asma, de la Facultad de Odontología de la Universidad Autónoma de Yucatán. Materiales y métodos: Estudio transversal, de casos y controles. Se incluyeron pacientes de entre 5 y 12 años, con diagnóstico de asma (casos) y pacientes sanos (controles), durante el período de agosto 2018 - febrero 2019. Fueron excluidos pacientes con enfermedades sistémicas. Se realizó una revisión intraoral evaluando caries, gingivitis, erosiones y bruxismo. Las diferencias entre grupos fueron determinadas mediante los estadísticos
$\mathrm{Chi}^{2}$ y Wilcoxon Sum-Rank. Resultados: La muestra incluyó un total de 228 pacientes, 109 femeninos y 119 masculinos. Se registraron 76 casos de pacientes asmáticos, con edad promedio de 7 años. No se encontró asociación entre el asma y caries dental $\left(\mathrm{Chi}^{2}=0,19, p=0,655, \mathrm{OR}=1,16\right)$; entre asma y gingivitis $(\mathrm{W}=5103, p=0,1492)$ y bruxismo $\left(\mathrm{Chi}^{2}=2,4, p=0,12, \mathrm{OR}=1,97\right)$. Únicamente, se encontró asociación significativa entre asma y erosión dental $\left(\mathrm{Chi}^{2}=5,3, p=0,02, \mathrm{OR}=2,01\right)$. Conclusiones: No se encontró relación entre el asma y la presencia/ausencia de caries, gingivitis y bruxismo. Los pacientes asmáticos presentaron 1,02 veces más posibilidades de presentar erosión dental en comparación con los pacientes sanos.

Palabras clave: Asma, caries dental, erosión dental, gingivitis, bruxismo.

\footnotetext{
${ }^{1}$ Licenciatura en Cirujano Dentista. Universidad Autónoma de Yucatán.

2 *Maestría en Investigación en Salud. Universidad Autónoma de Yucatán.martha.chuc@correo.uady.mx Calle 61 A \#492 Ax90 y Av. Itzáes, Centro. CP. 97000. Mérida, Yucatán, México. Teléfono: 9993153424

Doctorado en Ciencias (Materiales Poliméricos). Universidad Autónoma de Yucatán.

${ }^{4}$ Maestría en Investigación Educativa. Universidad Autónoma de Yucatán.

${ }^{5}$ Maestría en Odontología Infantil. Universidad Autónoma de Yucatán.

${ }^{6}$ Doctor en Ciencias. Universidad Autónoma de San Luis Potosí.
} 
Artigo original

\section{Manifestações orais em pacientes pediátricos com asma. Estudo de caso de controle}

\section{Resumo}

A asma afeta principalmente a população infantil de 3 à 14 anos. Crianças que sofrem de doenças respiratórias tendem a sofrer alterações na sua saúde bucal. Objetivo: Estabelecer as manifestações clínicas orais de pacientes pediátricos com asma, atendidos na Faculdade de Odontologia da Universidade Autônoma de Yucatán. Materiais e Métodos: Estudo transversal, casos e controles. Foram incluídas crianças com idades entre 5 e 12 anos, com diagnóstico de asma, e pacientes saudáveis que visitaram a instituição no período de agosto de 2018 à fevereiro de 2019. Foram excluídos os pacientes com doenças sistêmicas. Foi realizada uma revisão intraoral avaliando cárie, gengivite, erosões e bruxismo. As diferenças entre os grupos foram avaliadas usando as estatísticas
Chi ${ }^{2}$ e Wilcoxon Sum-Rank. Resultados: A amostra incluiu um total de 228 pacientes, sendo 109 do sexo feminino e 119 , do sexo masculino. Foram registrados 76 casos de pacientes asmáticos, com idade média de 7 anos. Não foi encontrada associação entre asma e cárie dentária $\left(\mathrm{Chi}^{2}=0,19\right.$ $p=0,655, \mathrm{OR}=1,16)$; bem como entre asma e gengivite $(\mathrm{W}=5103, \mathrm{p}=0,1492)$ e bruxismo $\left(\mathrm{Chi}^{2}=2,4, p=0,12, \mathrm{OR}=1,97\right)$. Somente uma associação significativa foi encontrada entre asma e erosão dentária $\left(\mathrm{Chi}^{2}=5,3\right.$, $p=0,02, \quad \mathrm{OR}=2,01)$. Conclusões: Não foi encontrada relação entre asma e presença / ausência de cárie, gengivite e bruxismo. Pacientes asmáticos tiveram 1,02 vezes mais chances de apresentar erosão dentária em comparação com pacientes saudáveis.

Palavra-chave: Asma, cárie dentária, erosão dentária, gengivite, bruxismo.

Original article

\section{Oral manifestations in pediatric patients with asthma. Case Control Study}

\begin{abstract}
Asthma is a chronic disease that affects the child population, mostly between 3 to 14 years of age. Children suffering from bronchial asthma usually present oral problems. Aim: To determine oral clinical manifestations of pediatric patients with asthma, treated at the Universidad Autónoma de Yucatán. Material and
\end{abstract}

methods: cross sectional, cases and control study. Children between 5 to 12 years old were included, with a diagnosis of asthma (cases) and healthy patients (control) who visited the institution from august 2018 to February 2019. Patients with systemic diseases were excluded. An intraoral review was realized evaluating dental caries, gingivitis, dental erosions and bruxism. Differences 
between groups were evaluated using the $\mathrm{Chi}^{2}$ and Wilcoxon Sum-Rank statistics. Results: The sample included a total of 228 patients, 109 female and 119 male. 76 cases of asthmatic patients were registered, an average age of 7 years. No association was found between asthma and dental caries $\left(\mathrm{Chi}^{2}=0.19 p=0.655\right.$, $\mathrm{OR}=1.16)$; there was no association between asthma and gingivitis ( $W=5103$, $p=0.1492)$ and bruxism $\left(\mathrm{Chi}^{2}=2.4, p=0.12\right.$,

\section{Introducción}

El asma, es una enfermedad heterogénea y multifactorial, caracterizada por la inflamación crónica de las vías aéreas y por la presencia de ataques recurrentes de disneas y sibilancias pudiendo variar de una persona a otra. La presencia de estos síntomas; pueden agravarse por las noches o al realizar actividades físicas. Con base en los signos y síntomas que el paciente refiera, esta enfermedad puede controlarse mediante corticoesteroides, entre otros medicamentos, que en su mayoría suelen ser inhalados. ${ }^{1}$

La Organización Mundial de la Salud (OMS) reporta que afecta a más de 235 millones de personas. ${ }^{2}$ La prevalencia de esta enfermedad es alta y creciente a nivel mundial en un $12 \% .^{1}$ Existen datos que informan que el $9 \%$ se da en niños de 7 años y el 5\% en adolescentes. ${ }^{3}$

Según el ISAAC (International Study of Asthma and Allergies in Childhood), Latinoamérica alcanza una prevalencia de asmáticos superior a 30\%, con una media en promedio del 17\% y con fluctuaciones
$\mathrm{OR}=1.97)$. Only significant association between asthma and dental erosion $\left(\mathrm{Chi}^{2}=5.3, p=0.02, \mathrm{OR}=2.01\right)$ was found. Conclusion: No association was found between asthma and the presence / absence of dental caries, gingivitis and bruxism. Asthmatic patients presented 1.02 more probability to have dental erosion compared to healthy patients.

Keywords: Asthma, dental caries, dental erosion, gingivitis, bruxism.

entre el 5\% en algunas ciudades de México y un $30 \%$ en Costa Rica. ${ }^{4}$ En México la prevalencia promedio de niños con asma es del $8 \%$, siendo mayor en las ciudades cercanas al Golfo de México. ${ }^{5}$

Estudios epidemiológicos de México han reportado que el estado de Yucatán ocupa los primeros lugares en incidencia de asma, debido al clima y a las condiciones ambientales de la región, siendo mayor en niños de 3 a 14 años. ${ }^{3}$

Los niños que padecen asma, o algún otro trastorno respiratorio, pueden presentar dificultades para mantener una adecuada salud oral. ${ }^{6}$ En la literatura actual, la asociación entre el asma y la presencia de manifestación bucales no ha sido concluyente. ${ }^{7}$ La mayoría de los estudios realizados en niños y adolescentes, señalan al asma como factor desencadenante de caries y enfermedad periodontal, con resultados discutibles. ${ }^{5}$ Estos estudios sugieren una asociación entre la medicación, por ejemplo, los antihistamínicos, antagonistas beta, inhalados y esteroides sistémicos, como factores etiológicos a estas manifestaciones orales en pacientes pediátricos con asma. ${ }^{8,9}$ 
Diversos autores mencionan que los pacientes asmáticos son más propensos a desarrollar respiración bucal, lo cual reduce el flujo salival y el $\mathrm{pH}$, también se menciona que los pacientes asmáticos pueden padecer de reflujo gastroesofágico; entre el 50\% y 60\% de los niños asmáticos sufren de síntomas de regurgitación ácida. ${ }^{6,8-11}$

La Academia Americana de Odontología Pediátrica, señala que los niños con enfermedades crónicas que consumen medicamentos pueden tener un mayor riesgo de padecer enfermedades bucodentales. ${ }^{6}$ Entre los cambios en la salud oral asociados al asma se encuentran: daños a tejidos duros del diente, como pueden ser caries, erosión dental y defectos en el esmalte, presencia de enfermedad periodontal, gingivitis, malposición dental, maloclusión, candidiasis, lengua fisurada, lengua geográfica, mordida abierta, entre otros..$^{5,6}$

En México, son pocos los estudios realizados al respecto. Por tal motivo, el objetivo del presente trabajo es establecer las manifestaciones clínicas bucales en pacientes con edades comprendidas entre 5 y 12 años, que padecen asma, atendidos en la Facultad de Odontología de la Universidad Autónoma de Yucatán (FOUADY).

\section{Materiales y métodos}

Previa aprobación por el comité de Investigación de la Facultad de Odontología de la Universidad Autónoma de Yucatán, bajo el proyecto de Investigación SISTPROY FODO-
2018-003, se solicitó autorización a cada paciente mediante la firma por parte del padre, madre o tutor de la Carta de Consentimiento informado, previa explicación y asentimiento de los menores.

Para este estudio de tipo observacional, descriptivo y transversal de casos y controles, fueron seleccionados pacientes pediátricos de entre 5 y 12 años de edad que acudieron a la FOUADY en el período de agosto 2018 a febrero 2019. La muestra fue tomada a conveniencia y de forma no probabilística incluyendo a todos los pacientes cuya historia médica refería padecer asma (casos). El grupo control estuvo conformado por pacientes sanos, que no padecían asma y acudieron para su atención en el período antes mencionado.

Se excluyeron pacientes con enfermedades sistémicas y niños asmáticos con otros problemas de salud.

Mediante un cuestionario formulado por 8 preguntas basado en el proyecto de asma titulado: "Estudio Internacional de Alergia y Asma en Niños, ISAAC", se confirmó el diagnóstico de asma. ${ }^{4,12-15}$

Se realizó la historia clínica del paciente, la cual comprendía de una serie de preguntas dirigidas a los padres o tutores, se obtuvo información acerca de los datos personales, evaluación general, antecedentes personales patológicos $\mathrm{y}$ práctica de higiene bucodental del niño.

\section{Examen clínico oral}

El examen de la cavidad oral se llevó a cabo por un observador en una unidad dental siguiendo la Norma Oficial Mexicana-013 (NOM-013) y fueron evaluadas las siguientes patologías bucales: 
Caries dental, se evaluó utilizando elíndice CPO-D y ceo- $\mathrm{d}^{16}$, gingivitis, por medio del índice $\mathrm{PMA}^{17}$, la erosión dental mediante el índice BEWE (Basic Erosive Wear Examination). ${ }^{18}$ El bruxismo, se determinó utilizando la Clasificación Internacional de los criterios de los trastornos del sueño (ICSD). ${ }^{19}$

Los datos obtenidos fueron capturados en una base de datos para ser analizados mediante estadística descriptiva e inferencial. La normalidad de los datos fue evaluada con Shapiro-Wilks y las diferencias entre grupos mediante la prueba estadística de Chi cuadrada y Wilcoxon Sum Rank Test, con un nivel de confianza del 95\%.

\section{Resultados}

Se estudiaron un total de 228 pacientes pediátricos, (109 femeninos y 119 masculinos). Los pacientes se asignaron en dos grupos, 76 pacientes asmáticos (grupo casos) y 152 pacientes que refirieron no padecer ninguna enfermedad (grupo control), en una proporción 1:2. (Tabla 1)

Se registraron 30 pacientes femeninos y 46

Tabla 1. Distribución de edad y sexo en los grupos.

\begin{tabular}{|l|c|c|c|}
\hline \multirow{2}{*}{ Pacientes } & Edad & \multicolumn{2}{|c|}{ Sexo Conteos (\%) } \\
\cline { 2 - 4 } & Media(sd) & Femenino & Masculino \\
\hline Casos & $7,605(1,98)$ & $30(39 \%)$ & $46(61 \%)$ \\
\hline Controles & $7,611(1,89)$ & $79(52 \%)$ & $73(28 \%)$ \\
\hline Valor de $p$ & $0,928^{*}$ & \multicolumn{2}{|c|}{$0,074^{* *}$} \\
\hline
\end{tabular}

*Wilcoxon

${ }^{* *}$ Chi-squared masculinos del grupo de casos. El grupo control con un total de 79 femeninos y 73 masculinos. La edad promedio de los pacientes fue de 7,6 años en ambos grupos.

\section{Caries}

El total de pacientes con caries fue de 176 (77\%), de los cuales 60 (79\%) pacientes corresponde al grupo de casos, distribuidos en 22 femeninos y 38 masculinos. En el grupo de control, 116 $(76 \%)$ pacientes presentaron caries, 61 femeninos y 55 masculinos. El puntaje obtenido del índice CPO-D fue de 4,6, que de acuerdo con el nivel de severidad se presentó en alto riesgo en ambos grupos de la muestra. La diferencia entre el grupo de casos y controles en el índice del CPO-D no fue significativa con Wilcoxon Sum Rank, $W=5916, p=0,764$. Adicionalmente, no existió asociación significativa entre el asma y la caries dental en la muestra estudiada $(p=0,655)$ (Tabla 2$)$.

\section{Gingivitis}

De los 76 pacientes del grupo de casos, el $83 \%$ presentó gingivitis a nivel de la encía papilar con un valor de PMA de 0,648. El sexo masculino fue el más frecuente con un $60 \%$. En el grupo control un $75 \%$ de

Tabla 2. Asociación entre asma y las variables caries, erosión y bruxismo.

\begin{tabular}{|l|c|c|c|c|}
\hline $\begin{array}{l}\text { Asociación } \\
\text { de asma } \\
\text { con }\end{array}$ & $\begin{array}{c}\text { Chi- } \\
\text { squared }\end{array}$ & $p$ & OR & IC 95\% \\
\hline Caries & 0,1993 & 0,6552 & 1,16 & $0,57-2,43$ \\
\hline Erosión & 5,361 & 0,0206 & 2,018 & $1,05-3,85$ \\
\hline Bruxismo & 2,42 & 0,12 & 1,97 & $0,74-5,16$ \\
\hline
\end{tabular}


los pacientes presentó gingivitis a nivel de la encía papilar con valor de PMA similar al grupo de casos $(0,648)$. El sexo femenino fue el más afectado con un $51 \%$. La diferencia en el valor de PMA entre el grupo casos y controles, no fue significativa con Wilcoxon Sum Rank, $p=0,149$.

\section{Erosión dental}

De la muestra total, $62(27 \%)$ pacientes presentaron erosión dental, 28 (37\%) pacientes del grupo de casos y 34 (22\%) pacientes del grupo control. Se observó una mayor presencia de erosiones en la arcada inferior y caras oclusales.

En cuanto a los niveles de riesgo de erosión, según el índice de BEWE, se encontró que el grupo control presentó un 6,5\% de pacientes con bajo riesgo y para los pacientes con asma, el 13,1\% presento bajo riesgo. Tanto para casos como controles, no hubo individuos en los niveles de riesgo medio y alto.

Por lo que concierne a la localización por arcada de las erosiones, en el grupo de casos en el maxilar superior se observó una mayor frecuencia en la zona anterior con un $70 \%$ en la superficie incisal y en el maxilar inferior (zona posterior) con un $83 \%$ en la superficie oclusal.

En el grupo control, el maxilar superior presentó mayor frecuencia de erosiones en la zona posterior y superficie oclusal con un $83 \%$. En el maxilar inferior se observó una mayor frecuencia en la zona posterior y en la superficie oclusal en un $100 \%$. Existe una asociación estadísticamente significativa entre el asma y la erosión dental con Chi-squared, $p=0,021$, OR=
2,018 IC= 1,05, 3,85 (Tabla 2), lo que indica que los niños con asma tienen 1,02 veces más probabilidad de presentar erosión dental que los niños que no tienen asma.

\section{Bruxismo}

En el grupo de casos, 11 (14\%) pacientes fueron diagnosticados como bruxistas y en el grupo control 12 pacientes (8\%). En este sentido, no existe asociación significativa entre asma y bruxismo con Chi-squared, $\mathrm{p}>0,05$ (Tabla 2).

\section{Discusión}

Actualmente el asma se considera un problema de salud pública, la prevalencia de esta enfermedad a nivel mundial va en aumento, sobre todo en infantes. ${ }^{1}$ Los estudios realizados entre el asma y las repercusiones en la salud bucal son limitados y con resultados muy variados, debido a que diferentes autores mencionan asociación con la enfermedad, mientras otros plantean que no existe relación. ${ }^{7}$

El presente estudio se conformó de 76 pacientes con diagnóstico de asma, siendo la mayoría hombres. Lo cual coincide con estudios realizados en pacientes asmáticos, donde se reporta una mayor prevalencia en el sexo masculino; el cual se considera un factor de riesgo, ${ }^{5}$ debido a que tiene una prevalencia alta antes de los 14 años, esto puede deberse a que el tamaño de los pulmones es menor en los hombres que en las mujeres al momento del nacimiento. ${ }^{12}$

La caries dental es una enfermedad crónica y la más frecuente de la infancia, 
afecta entre el $60 \%$ y $90 \%$ de la población infantil. ${ }^{20}$ A pesar de que numerosos estudios han relacionado la caries dental en pacientes con asma, ${ }^{21-23}$ aún no existe suficiente evidencia de su asociación.

En la presente investigación, no se encontró una asociación estadísticamente significativa en el número de dientes afectados por caries en los pacientes asmáticos en comparación con los pacientes sanos.

Los resultados de nuestro estudio son consistentes con los hallazgos presentados por diversos autores, en los cuales no se relaciona al asma con la presencia o el riesgo de padecer caries dental. ${ }^{13,14,24}$ Sin embargo existen otras publicaciones que reportan hallazgos de un alto valor del índice CPO-D en niños asmáticos. ${ }^{13}$

A pesar de que en nuestro estudio no se encontraron diferencias en número, una gran cantidad de pacientes asmáticos (79\%), sí presentaron lesiones de caries. Lo anterior podría justificarse por la medicación que consumen dichos pacientes, ya que se afirma que este tipo de medicamentos desempeña un papel importante en el desarrollo de caries dental, reduciendo el flujo salival, una gran proporción del medicamento inhalado se retiene en la orofaringe, éste contiene azucares y saborizantes para que pueda ser tolerado por el paciente. ${ }^{13}$ Yadav, et al. observaron en sus resultados que los niños asmáticos sufrían de una carencia en su estado de salud oral debido a una alta incidencia de caries, gingivitis e higiene oral comprometida, atribuyéndoselo al tipo de medicación que consumen dichos pacientes. ${ }^{25}$

Hassanpour et al. compararon la frecuencia de caries dental mediante el índice CPO-D, en pacientes asmáticos con tratamiento de corticoesteroides inhalados. Como resultado observaron una mayor frecuencia de caries dental en pacientes con asma con un CPO-D de 0,71, reportando una diferencia estadísticamente significativa comparada con el grupo control. Los pacientes asmáticos referían en su historia médica haber consumido corticoesteroides en aerosol durante 2 años. ${ }^{21}$

Renzende et al., señala que los niños entre 6 y 12 años con asma sin medicación no presentaron una asociación estadísticamente significativa, sin embargo, hubo una asociación entre el asma y la medicación con salbutamol, lo cual presento un aumento del $32 \%$ de caries dental. ${ }^{22}$

Chellaih et al. mencionan que la puntuación del CPO-D fue mayor en el grupo de casos, estudiaron pacientes con medicación de corticoesteroides inhalados a diario por más de 2 años, siendo 6 veces más probable el desarrollo de caries dental en los asmáticos. ${ }^{23}$

A diferencia de nuestra investigación, la mayoría de los estudios que presentaron una asociación son longitudinales y evalúan una determinada medicación, lo que podría explicar la diferencia en los resultados obtenidos.

El control de la placa dental es la base para una correcta higiene bucal, se menciona que los niños con asma tienen valores más altos de índice de placa, provocando la aparición de enfermedades periodontales. ${ }^{26}$

Evaluamos la presencia de gingivitis donde se reportó un $83 \%$ a nivel de la encía papilar sin diferencias entre el asma y la 
gingivitis, siendo estos resultados acordes a lo reportado en la literatura. ${ }^{13,14,27}$

Sin embargo, Yaghobee et al. demostraron en su estudio diferencias significativas entre la presencia de gingivitis en los grupos con asma y control. Mencionan que estas diferencias pudieron ser ocasionadas por los efectos de la medicación sobre la microbiota bucal, como lo es la caída del $\mathrm{pH}$, llegando a valores muy bajos, lo cual aumenta la actividad de las bacterias patológicas en la placa gingival. ${ }^{28} \mathrm{La}$ disminución en el $\mathrm{pH}$ de la saliva y la placa dentobacteriana en los asmáticos es causada por el fármaco y no por la enfermedad. ${ }^{23}$

Arafa et al., obtienen como resultado que los niños asmáticos tenían una puntuación significativamente mayor de inflamación gingival que los controles, donde la condición gingival se deteriora a medida que la condición de asma se vuelve más severa. ${ }^{29}$

La presencia de gingivitis en pacientes asmáticos puede atribuirse a una respuesta inmune alterada, y a la tendencia de respirar por la boca causando deshidratación de la mucosa alveolar, debido a la presencia de ataques asmáticos agudos. ${ }^{30}$

A pesar de que nuestros resultados no demostraron una asociación entre asma y gingivitis, los pacientes asmáticos presentaron gingivitis en un $83 \%$, esto puede explicarse por el tipo de respiración que manifiestan, ya que la mayoría suele respirar por la boca a causa de la obstrucción nasal que padecen, sobre todo cuando sufren de una crisis asmática o un cambio climático, disminuyendo el flujo salival causando sequedad de la mucosa oral en el sector anterior del maxilar superior, favoreciendo la colonización bacteriana y por ende el crecimiento de la placa dentobacteriana, lo que conllevaría a desarrollar gingivitis. ${ }^{31}$

La disminución de la cantidad de saliva y del pH se ha detectado en niños asmáticos después del uso de inhaladores con dosis medidas, algunos de ellos contienen lactosa como vehículo, haciendo que los valores de $\mathrm{pH}$ en la mayoría de estos polvos estén a menos de 5,5; lo que asociado a una tasa de flujo salival reducido puede ser un factor de riesgo para la erosión dental. ${ }^{27}$

Respecto a la erosión dental, se evidenció una asociación estadísticamente significativa con el asma, con una presencia del $37 \%$ de erosiones en pacientes asmáticos, lo cual coincide con el estudio realizado por Manish Jain et al., quienes reportan un nivel de erosión significativamente mayor en asmáticos. Plantean que la mayoría de los medicamentos para el asma son ácidos, disminuyen la tasa de flujo salival y promueven el reflujo gastroesofágico lo que representan una amenaza erosiva para la dentición. Sin embargo, también reportan que la prevalencia de erosión en los pacientes asmáticos puede deberse a la dieta y a la preferencia de consumir más refrescos y bebidas carbonatadas. ${ }^{32}$

Los pacientes con asma presentan un mayor riesgo a sufrir erosión dental, posiblemente por los por efectos de la medicación que causan reducción en el flujo salival. ${ }^{13}$

El bruxismo del sueño se ha relacionado con la obstrucción parcial prolongada 
o completa e intermitente de las vías aéreas. $^{33}$ El asma puede ser un factor etiológico para el desarrollo del bruxismo, debido a la obstrucción nasal que produce dicha enfermedad.

En los resultados de nuestra investigación, un $14 \%$ de la muestra de pacientes asmáticos fueron diagnosticados como bruxistas, y se determinó que no existe una relación estadísticamente significativa con el asma en esta muestra. Sin embargo, se ha señalado que los pacientes asmáticos, tienen un mayor riesgo a desarrollar bruxismo del sueño. ${ }^{34} \mathrm{Di}$ Francesco et al. sugieren que los niños con vías respiratorias obstruidas tienden a empujar la mandíbula hacia adelante y hacia abajo para mejorar el paso del aire, estimulando a los receptores de las vías respiratorias superiores a intensificar el tono, provocando el bruxismo. ${ }^{35,36}$ A pesar de lo descrito, no hay suficiente evidencia en la que se explique un resultado congruente de la existencia de una asociación con el asma.
Estos resultados discrepantes podrían explicarse por la utilización de diversas escalas para medir las variables evaluadas en el presente estudio, que si bien, fueron realizadas como están descritas originalmente, no fue posible realizar previa calibración del examinador, esto podría influir en la validez externa de nuestro estudio.

\section{Conclusiones}

El asma no presentó asociación estadísticamente significativa con el aumento en las lesiones cariosas, gingivitis y bruxismo en este estudio de casos/controles. Se observó una asociación estadísticamente significativa con la erosión dental, indicando que los niños con asma tienen más probabilidad de presentar dicha patología.

\section{Referencias bibliográficas}

1. López G, Huerta J. Asma perspectiva actual. 1a ed. Tovar GG; 2016.

2. OMS. Asma. Agosto 2017. Disponible en: URL: https://www.who.int/es/news-room/fact-sheets/detail/ asthma

3. Bacab M. Prevalencia de asma aguda en niños y adolescentes de Mérida, Yucatán, México. Rev Alerg México. 2009;56(1):3-8.

4. Ocampo J, Gaviria R, Sánchez J. Prevalecia del asma en América Latina. Mirada crítica a partir del ISAAC y otros estudios. Rev Alerg Mex. 2017;64(2):188-197.

5. Navarrete E, Sienra JJL, Pozo CF. Asma en pediatría. Rev la Fac Med la UNAM. 2016;59(4):5-15.

6. Widmer RP. Oral health of children with respiratory diseases. Paediatr Respir Rev. 2010;11(4):226-32.

7. Wierchola B, Emerich K, Adamowicz K. The association between bronchial asthma and dental caries in

8. Lareneas D, et al. Mexican Asthma Guidelines: GUIMA 2017 Rev Alerg Mex Guía Mex del Asma. 2017;64(1):11-128.

9. Samec T, Amaechi BT, Battelino T, Krivec U, Jan J. Influence of anti-asthmatic medications on dental caries in children in Slovenia. Int J Paediatr Dent. 2013;23(3):188-96.

10. Marchena LR, Fernandez C. Tratamiento Multidisciplinar Del Asma Infantil. REDOE. 2018;1-5.

11. Sivasithamparam K, Young WG, Jirattanasopa V, Priest J, Khan F, Harbrow D, et al. Dental erosion in asthma: a case-control study from south east Queensland. Aust Dent J. 2002;47(4):298-303. 
12. García S, Pérez S. Asma: concepto, fisiopatología, diagnóstico y clasificación. Pediatr Integral. 2012;17(2):117-30.

13. Qasem FA, Awad SM, Shalan HM, El-Desoky T. Assessment of Oral Health Status in a Group of Asthmatic Children. Oral Health Dent Manag. 2018;17(1):1-5.

14. Ferrazzano GF, Sangianantoni G, Cantile T, Amato I, Ingenito A NP. Dental health in asthmatic children: a Douth Italy study. J Dent Child. 2012;79(3):170-5.

15. Turkistani JM, Farsi N, Almushayt A, Alaki S. Caries Experience in Asthmatic Children: A Review of Literature. J Clin Pediatr Dent. 2015;35(1):1-8.

16. García-Cortés JO, et al. Experiencia, prevalencia, severidad, necesidades de tratamiento para caries dental e índice de cuidados en adolescentes y adultos jóvenes mexicanos. Rev Invest Clin. 2014;66(6):505-511.

17. Newman M, Takei H, Klokkevold P, Carranza F. Periodontologia clinica de Carrranza. $11^{\circ}$ ed. Amolca. 2014.

18. Bartlett D, Ganss C, Lussi A. Basic Erosive Wear Examination (BEWE): a new scoring system for scientific and clinical needs. Clin Oral Invest. 2008;12(1):S65-S68.

19. Amato JN, Tuon RA, Castelo PM, Gaviao MBD, Barbosa TDS. Assessment of sleep bruxism, orthodontic treatment need, orofacial dysfunctions and salivary biomarkers in asthmatic children. Arch Oral Biol. 2015;60(5):698-7.

20. Morales L, Gómez W. Caries dental y sus consecuencias clínicas relacionadas al impacto en la calidad de vida de preescolares de una escuela estatal. Rev. Estomatol Herediana. 2019;29(1):17-29.

21. Hassanpour K, Tehrani H, Goudarzian M, Beihaghi S, Ebrahimi M, Amiri P. Comparison of the frequency of dental caries in asthmatic children under treatment with inhaled corticosteroids and healthy children in Sabzevar in 2017-2018. Electron J Gen Med. 2019;16(2):1-6.

22. Rezende et al. Asthma and oral changes in children: Associated factors in a community of southern Brazil. Int J Paediatr Dent. 2019;29:456-463.

23. Chellaih P, Sivadas G, Chintu S, Vedam VKV, Arunachalam R, Sarsu M. Effect of anti-asthmatic grugs on dental health: A comparative Study. J Pharm Bioallied Sci. 2016;8(1):77-80.

24. Ehsani S, Moin M, Meighani G, Pourhashemi SJ, Khayatpisheh H, Yarahmadi N. Oral health status in preschool asthmatic children in Iran. Iran J Allergy, Asthma Immunol. 2013;12(3):254-61.

25. Yadav K, Saha S, Singh S, et al. Oral health status and treatment need of asthmatic children aged 6-12 years in Lucknow. J Indian Assoc Public Heal Dent. 2017;15(2):122-6.

26. Davidovié B, et al. Asthma and periodontal health in children. Vojnosanit Pregl. 2018;75(12): 1202-1208.

27. Eloot AK, Vanobbergen JN, Baets FDE, Martens LC. Oral health and habits in children with asthma related to severity and durantion of condition. EJPD. 2004;5(4):210-5.

28. Yaghobee S, Paknejad M, Khorsand A. Association between Asthma and Periodontal Disease. J Dent (Tehran). 2008;5(2):47-51.

29. Arafa A, Aldahlawi S, Fathi A. Assessment of the oral health status of asthmatic children. Eur J Dent. 2017;11(3):357-63.

30. Shashikiran N, Reddy VVS, Raju PK. Effect of antiasthmatic medication on dental disease: Dental caries and periodontal disease. J Indian Soc Pedod Prev Dent. 2007;25(2):65-8.

31. Ramos J, Ramírez E, Vázquez EM, Vázquez F. Repercusiones en la salud bucodental asociadas con el asma en niños de 6 a 12 años de edad. Rev Alerg Mex. 2017;64(3):270-76.

32. Jain M, Mathur A, Sawla L, et al. Prevalence of dental erosion among asthmatic patients in India. Rev clín pesq odontol. 2009;5(3):247-54.

33. Hermida L, Restrepo C. Bruxismo del sueño y sídrome de apnea obstructiva del sueño en niños. Revisión narrativa. Revista de Odontopediatría Latinoamericana. 2016;6(2):99-107.

34. American Academy of Sleep Medicine. The International Classification of sleep disorders: Diagnostic and coding manual. 2nd ed. American Academy of Sleep Medicine; 2005.

35. Bolaños E, Gonzales E, Del Castillo E. El bruxismo infantil. Odontol Pediátrica. 2002;10(3):135-41.

36. Bortoletto C, Marques A, Ferrari RA, Fernandes KP, Bussadori S, Motta L. Association between respiratory problems and dental caries in children with bruxism. Indian J Dent Res. 2014;25(1):9.

Recibido: : $10 / 06 / 2020$

Aceptado: 13/08/2020

Correspondencia: Martha Gabriela Chuc Gamboa, correo: martha.chuc@correo.uady.mx 